\title{
An Efficient Multiple Particle Filter Based on the Variational Bayesian Approach
}

\author{
Boujemaa Ait-El-Fquih \\ Division of Computer, Electrical \\ and Mathematical Science \& Engineering \\ King Abdullah University of Science and Technology \\ Thuwal, Saudi Arabia \\ Email: boujemaa.aitelfquih@kaust.edu.sa
}

\author{
Ibrahim Hoteit \\ Division of Computer, Electrical \\ and Mathematical Science \& Engineering \\ King Abdullah University of Science and Technology \\ Thuwal, Saudi Arabia \\ Email: ibrahim.hoteit@kaust.edu.sa
}

\begin{abstract}
This paper addresses the filtering problem in largedimensional systems, in which conventional particle filters (PFs) remain computationally prohibitive owing to the large number of particles needed to obtain reasonable performances. To overcome this drawback, a class of multiple particle filters (MPFs) has been recently introduced in which the state-space is split into low-dimensional subspaces, and then a separate PF is applied to each subspace. In this paper, we adopt the variational Bayesian (VB) approach to propose a new MPF, the VBMPF. The proposed filter is computationally more efficient since the propagation of each particle requires generating one (new) particle only, while in the standard MPFs a set of (children) particles needs to be generated. In a numerical test, the proposed VBMPF behaves better than the PF and MPF.
\end{abstract}

\section{INTRODUCTION}

Consider a discrete time state-space dynamical system:

$$
\left\{\begin{aligned}
\mathbf{x}_{n+1} & =\mathbf{f}_{n}\left(\mathbf{x}_{n}\right)+\mathbf{u}_{n} \\
\mathbf{y}_{n} & =\mathbf{H}_{n} \mathbf{x}_{n}+\mathbf{v}_{n}
\end{aligned}\right.
$$

in which $\mathbf{x}_{n} \in \mathbb{R}^{n_{\mathbf{x}}}$ and $\mathbf{y}_{n} \in \mathbb{R}^{n_{\mathbf{y}}}$ denote the system state and the observation at time instant $n$, respectively. $\mathbf{f}_{n}$ is a nonlinear transition operator integrating the system state from time $n$ to $n+1, \mathbf{H}_{n}$ a linear observational operator at time $n$. The input noise, $\mathbf{u}=\left\{\mathbf{u}_{n}\right\}_{n \in \mathbb{N}}$, and the observation noise, $\mathbf{v}=$ $\left\{\mathbf{v}_{n}\right\}_{n \in \mathbb{N}}$, are assumed to be independent, jointly independent, and independent of the initial state, $\mathbf{x}_{0}$. Throughout this paper, $\mathbf{y}_{0: n}$ denotes the shorthand of the available observation set, $\left\{\mathbf{y}_{0}, \mathbf{y}_{1}, \cdots, \mathbf{y}_{n}\right\}$. Let also $p\left(\mathbf{x}_{n}\right)$ and $p\left(\mathbf{x}_{n} \mid \mathbf{y}_{0: m}\right)$ denote the probability density function (pdf) of $\mathbf{x}_{n}$ and the pdf of $\mathbf{x}_{n}$ conditional on $\mathbf{y}_{0: m}$, respectively; the other pdfs are defined similarly.

We focus on the Bayesian filtering problem which consists in computing the so-called filtering pdf, $p\left(\mathbf{x}_{n} \mid \mathbf{y}_{0: n}\right)$, for $n=0,1, \cdots$. Based on this pdf, any estimate can then be computed as for instance the a posteriori mean (AM) which minimizes the a posteriori mean square error (MSE). An efficient computation of the filtering pdf is possible based on the probabilistic Markovian / independence properties of system (1). Indeed, it has been shown that such a system is indeed a hidden Markov chain (HMC) model. In other words, the state process, $\mathbf{x}$, evolves following a Markov chain with transition density, $p\left(\mathbf{x}_{k} \mid \mathbf{x}_{k-1}\right)$; the observed process, $\mathbf{y}$, is independent conditionally on the state process; and the observation at any time $k, \mathbf{y}_{k}$, depends on the state process through the state at the same time, $\mathbf{x}_{k}$, via the likelihood, $p\left(\mathbf{y}_{k} \mid \mathbf{x}_{k}\right)$. In the HMC framework, one can recursively compute the filtering pdf following two steps [1]:

- Propagation (or prediction) step, which uses the transition pdf of HMC, $p\left(\mathbf{x}_{n} \mid \mathbf{x}_{n-1}\right)$, to compute the prediction pdf:

$$
p\left(\mathbf{x}_{n} \mid \mathbf{y}_{0: n-1}\right)=\int p\left(\mathbf{x}_{n} \mid \mathbf{x}_{n-1}\right) p\left(\mathbf{x}_{n-1} \mid \mathbf{y}_{0: n-1}\right) d \mathbf{x}_{n-1} .
$$

- Update (or filtering) step, in which the likelihood, $p\left(\mathbf{y}_{n} \mid \mathbf{x}_{n}\right)$, is used to update the prediction pdf via the Bayes' rule:

$$
p\left(\mathbf{x}_{n} \mid \mathbf{y}_{0: n}\right)=\frac{p\left(\mathbf{y}_{n} \mid \mathbf{x}_{n}\right) p\left(\mathbf{x}_{n} \mid \mathbf{y}_{0: n-1}\right)}{\int p\left(\mathbf{y}_{n} \mid \mathbf{x}_{n}\right) p\left(\mathbf{x}_{n} \mid \mathbf{y}_{0: n-1}\right) d \mathbf{x}_{n}} .
$$

In practice, unless the HMC is linear and Gaussian, say, $\mathbf{f}_{n}$ in (1) is linear, and $\mathbf{u}_{n}$ and $\mathbf{v}_{n}$ are Gaussian, an analytical computation of the integrals in (2)-(3) is rarely possible [1], [2]. Therefore, many numerical methods approximating these integrals have been developed [1]-[6]. Among the most popular numerical methods are the Particle Filters (PFs), which aim at propagating a Monte Carlo (MC) approximation of $p\left(d \mathbf{x}_{n} \mid \mathbf{y}_{0: n}\right)$, and subsequently a MC approximation of the AM estimate [1], [2], [4]-[6]. However, it is well-known that $\mathrm{PFs}$, in their generic form, suffer from weights degeneracy in the sense that after some filtering cycles, the weights of all particles, except one, become almost zero, which significantly degrades the estimation performance. A number of judicious choices of importance densities, along with various resampling strategies have been proposed in an attempt to tackle this issue [2], [6]. Although many of these have provided satisfactory results in a number of low-dimensional state-space applications [4], [6], they nevertheless remain inefficient in the cases of very large dimensional system [7]-[11]. This is mainly due to the fact that the number of particles required to sufficiently sample the state-space needs to be very large; in some special situations it has been shown that the required number of particles scales exponentially with the system dimension (see e.g. [10]). 
A class of Multiple PFs (MPFs) has been recently proposed for efficient implementation of the PF [7]-[9], [12]-[14]. The idea is to split the state-space into $K$ subspaces of smaller dimensions, assuming the associated state partitions to be independent (a posteriori), so that a PF is then applied to each subspace. In this paper, we resort to the Variational Bayesian (VB) approach to introduce a new MPF, the VBMPF, which we derive by combining VB-like density approximations with MC-like approximations. Instead of directly assuming that the posterior pdf of the system state is separable, this pdf is first approximated by a product of independent marginal pdfs based on the Kullback-Leibler (KL) divergence minimization criteria (VB approximation stage) [15]-[17]. Each VB-like marginal distribution is then sampled as in the standard $\mathrm{PF}$ [5]. Each PF component of the VBMPF uses particles from the other components, as in the MPF, but following a different mechanism. The idea of using the VB approach to split the state-space has been first used in high-dimensional linear Gaussian systems, leading to fast Kalman-based algorithms [18], [19].

This paper is organized as follows. Section II describes the generic algorithm that recursively computes VB approximations of the filtering pdf. Its practical derivation using two classical random sampling strategies, leading to the VBMPF algorithm, will then be the focus of Section III. In Section IV, numerical simulations are performed comparing the performances of the proposed VBMPF w.r.t. the standard PF and a MPF. Concluding remarks are finally given in Section V.

\section{THE GENERIC ALGORITHM}

Let the state vector, $\mathbf{x}_{n}$, be split into $K$ partitions, $\mathbf{x}_{n}^{1}, \mathbf{x}_{n}^{2}, \cdots, \mathbf{x}_{n}^{K}$, of the same dimension, $n_{\mathbf{x}^{k}}=\frac{n_{\mathbf{x}}}{K} \in \mathbb{N}$. The extension of the proposed scheme to the more general case of different dimensions, $n_{\mathbf{x}^{k}}$, is trivial. A separable VB approximation, $q\left(\mathbf{x}_{n} \mid \mathbf{y}_{0: n}\right) \stackrel{\text { def }}{=} \prod_{k=1}^{K} q\left(\mathbf{x}_{n}^{k} \mid \mathbf{y}_{0: n}\right)$, of the filtering pdf, $p\left(\mathbf{x}_{n} \mid \mathbf{y}_{0: n}\right)$, is obtained under the criteria,

$$
q\left(\mathbf{x}_{n} \mid \mathbf{y}_{0: n}\right)=\underset{\prod_{k} \tilde{p}\left(\mathbf{x}_{n}^{k} \mid \mathbf{y}_{0: n}\right)}{\operatorname{argmin}} \operatorname{KL}\left(\prod_{k=1}^{K} \tilde{p}\left(\mathbf{x}_{n}^{k} \mid \mathbf{y}_{0: n}\right)|| p\left(\mathbf{x}_{n} \mid \mathbf{y}_{0: n}\right)\right),
$$

where each marginal $q\left(\mathbf{x}_{n}^{k} \mid \mathbf{y}_{0: n}\right)$ is given by [17], [18]:

$$
q\left(\mathbf{x}_{n}^{k} \mid \mathbf{y}_{0: n}\right) \propto \exp \left(\mathbb{E}_{q\left(\mathbf{x}_{n}^{k-} \mid \mathbf{y}_{0: n}\right)}\left[\ln \left(p\left(\mathbf{x}_{n}, \mathbf{y}_{0: n}\right)\right)\right]\right),
$$

with $\mathbf{x}_{n}^{k^{-}}$denotes the complement of $\mathbf{x}_{n}^{k}$ in the vector $\mathbf{x}_{n}$, and $\mathbb{E}_{p(\mathbf{x})}[f(\mathbf{x})]$ the expected value of a function $f(\mathbf{x})$ w.r.t. the distribution $p(\mathbf{x})$. Eq. (5) points to the fact that although the approximated marginal pdf of $\mathbf{x}_{n}^{k}, q\left(\mathbf{x}_{n}^{k} \mid \mathbf{y}_{0: n}\right)$, is independent of those of the other components $\mathbf{x}_{n}^{k^{-}}, q\left(\mathbf{x}_{n}^{k^{-}} \mid \mathbf{y}_{0: n}\right)$, it nevertheless remains dependent on the expected value of $\ln \left[p\left(\mathbf{x}_{n}, \mathbf{y}_{0: n}\right)\right]$ w.r.t. $q\left(\mathbf{x}_{n}^{k-} \mid \mathbf{y}_{0: n}\right)$. In other words, the "enforced" independence on the marginal pdfs is partially compensated by a functional dependence involving expectations w.r.t. these pdfs. Below we propose a recursive algorithm to compute the solutions (5) based on (2)-(3) in two steps.

\section{A. Prediction step}

Starting at time $n-1$ from a VB-like approximation,

$$
p\left(\mathbf{x}_{n-1} \mid \mathbf{y}_{0: n-1}\right) \approx \prod_{k=1}^{K} q\left(\mathbf{x}_{n-1}^{k} \mid \mathbf{y}_{0: n-1}\right),
$$

we aim at computing a separable approximation of the prediction pdf, $p\left(\mathbf{x}_{n} \mid \mathbf{y}_{0: n-1}\right)$, using (2). Let assume the following factorization of the HMC transition pdf:

$$
p\left(\mathbf{x}_{n} \mid \mathbf{x}_{n-1}\right)=\prod_{k=1}^{K} p\left(\mathbf{x}_{n}^{k} \mid \mathbf{x}_{n-1}\right) .
$$

Inserting (6) and (7) in (2), one obtains,

$p\left(\mathbf{x}_{n} \mid \mathbf{y}_{0: n-1}\right) \approx \int \prod_{k=1}^{K} p\left(\mathbf{x}_{n}^{k} \mid \mathbf{x}_{n-1}\right) q\left(\mathbf{x}_{n-1}^{k} \mid \mathbf{y}_{0: n-1}\right) d \mathbf{x}_{n-1}$.

However, the approximation (8) is not separable, i.e., it is not a product of $K$ marginal pdfs of $\mathbf{x}_{n}^{k}$ given $\mathbf{y}_{0: n-1}$, unless the marginal processes, $\mathbf{x}^{k}=\left\{\mathbf{x}_{n}^{k}\right\}_{n}$, are independent Markov chains $\left(p\left(\mathbf{x}_{n}^{k} \mid \mathbf{x}_{0: n-1}\right)=p\left(\mathbf{x}_{n}^{k} \mid \mathbf{x}_{0: n-1}^{k}\right)=p\left(\mathbf{x}_{n}^{k} \mid \mathbf{x}_{n-1}^{k}\right)\right.$; see [18], [19] for the linear Gaussian case). Moreover, using this approximation in the filtering step (3) does not guarantee a separable approximation of $p\left(\mathbf{x}_{n} \mid \mathbf{y}_{0: n}\right)$ even when the approximation of $p\left(\mathbf{x}_{n-1} \mid \mathbf{y}_{0: n-1}\right)$ (6) is separable. One way to tackle this issue is to approximate the marginal transition pdf, $p\left(\mathbf{x}_{n}^{k} \mid \mathbf{x}_{n-1}\right)$ (involved in (8)), which is indeed equal to $p\left(\mathbf{x}_{n}^{k} \mid \mathbf{x}_{n-1}, \mathbf{y}_{0: n-1}\right)$, by a pdf of the form, $q\left(\mathbf{x}_{n}^{k} \mid \mathbf{x}_{n-1}^{k}, \mathbf{y}_{0: n-1}\right)$. The idea is then to drop the dependence between $\mathbf{x}_{n}^{k}$ and $\mathbf{x}_{n-1}^{k-}$ conditionally on $\left(\mathbf{x}_{n-1}^{k}, \mathbf{y}_{0: n-1}\right)$, for $k=1,2, \cdots, K$. This can be done using the VB approach by approximating $p\left(\mathbf{x}_{n}^{k}, \mathbf{x}_{n-1}^{k-} \mid \mathbf{x}_{n-1}^{k}, \mathbf{y}_{0: n-1}\right)$ as

$$
\begin{aligned}
p\left(\mathbf{x}_{n}^{k}, \mathbf{x}_{n-1}^{k-} \mid \mathbf{x}_{n-1}^{k}, \mathbf{y}_{0: n-1}\right) & \approx q\left(\mathbf{x}_{n}^{k} \mid \mathbf{x}_{n-1}^{k}, \mathbf{y}_{0: n-1}\right) \\
& \times \underbrace{q\left(\mathbf{x}_{n-1}^{k-} \mid \mathbf{x}_{n-1}^{k}, \mathbf{y}_{0: n-1}\right)}_{q\left(\mathbf{x}_{n-1}^{k-} \mid \mathbf{y}_{0: n-1}\right)(\operatorname{see}(6))} .
\end{aligned}
$$

Similarly to (5), one obtains,

$q\left(\mathbf{x}_{n}^{k} \mid \mathbf{x}_{n-1}^{k}, \mathbf{y}_{0: n-1}\right) \propto \exp \left(\mathbb{E}_{q\left(\mathbf{x}_{n-1}^{k^{-}} \mid \mathbf{y}_{0: n-1}\right)}\left[\ln \left(p\left(\mathbf{x}_{n}^{k} \mid \mathbf{x}_{n-1}\right)\right)\right]\right)$.

Replacing in (8) $p\left(\mathbf{x}_{n}^{k} \mid \mathbf{x}_{n-1}\right)$ by its approximation (10), one eventually obtains a separable approximation of the joint prediction pdf:

$$
p\left(\mathbf{x}_{n} \mid \mathbf{y}_{0: n-1}\right) \approx \prod_{k=1}^{K} q\left(\mathbf{x}_{n}^{k} \mid \mathbf{y}_{0: n-1}\right),
$$

with

$q\left(\mathbf{x}_{n}^{k} \mid \mathbf{y}_{0: n-1}\right)=\int q\left(\mathbf{x}_{n}^{k} \mid \mathbf{x}_{n-1}^{k}, \mathbf{y}_{0: n-1}\right) q\left(\mathbf{x}_{n-1}^{k} \mid \mathbf{y}_{0: n-1}\right) d \mathbf{x}_{n-1}^{k}$. 


\section{B. Filtering step}

This section considers the computation, for each $k$, of the VB-like marginal filtering pdf, $q\left(\mathbf{x}_{n}^{k} \mid \mathbf{y}_{0: n}\right)$, given in (5), from the VB-like marginal prediction pdf, $q\left(\mathbf{x}_{n}^{k} \mid \mathbf{y}_{0: n-1}\right)$. Inserting in (5) the factorization,

$$
p\left(\mathbf{x}_{n}, \mathbf{y}_{0: n}\right) \propto p\left(\mathbf{y}_{n} \mid \mathbf{x}_{n}\right) p\left(\mathbf{x}_{n} \mid \mathbf{y}_{0: n-1}\right),
$$

then using (11) one obtains ${ }^{1}$,

$$
q\left(\mathbf{x}_{n}^{k} \mid \mathbf{y}_{0: n}\right) \propto q\left(\mathbf{x}_{n}^{k} \mid \mathbf{y}_{0: n-1}\right) \times \underbrace{\exp \left(\mathbb{E}_{q\left(\mathbf{x}_{n}^{k-} \mid \mathbf{y}_{0: n}\right)}\left[\ln \left(p\left(\mathbf{y}_{n} \mid \mathbf{x}_{n}\right)\right]\right)\right.}_{\mathcal{L}\left(\mathbf{x}_{n}^{k}\right)} .
$$

The computation of $q\left(\mathbf{x}_{n}^{k} \mid \mathbf{y}_{0: n}\right)$ using (13) requires the knowledge of $q\left(\mathbf{x}_{n}^{k-} \mid \mathbf{y}_{0: n}\right)$. However, $q\left(\mathbf{x}_{n}^{k-} \mid \mathbf{y}_{0: n}\right)$ are not known, which makes it impossible to derive an analytical solution of (13). Similarly to [17], [18], one may circumvent this issue by proceeding with iterations of (13) for all $k$, starting from the initialization $q\left(\mathbf{x}_{n}^{1-} \mid \mathbf{y}_{0: n}\right)=q\left(\mathbf{x}_{n}^{1-} \mid \mathbf{y}_{0: n-1}\right)$, to approximate the VB marginal filtering pdfs of interest.

\section{Practical DERIVATION}

We focus here on the practical derivation of the generic algorithm described above. Lets assume $\mathbf{x}_{0} \sim \mathcal{N}\left(\hat{\mathbf{x}}_{0}, \mathbf{P}_{0}\right)$, $\mathbf{u}_{n} \sim \mathcal{N}\left(\mathbf{0}, \mathbf{Q}_{n}\right)$, and $\mathbf{v}_{n} \sim \mathcal{N}\left(\mathbf{0}, \mathbf{R}_{n}\right)$, with $\mathbf{P}_{0}, \mathbf{Q}_{n}$ and $\mathbf{R}_{n}$ are positive definite matrices; $\mathbf{0}$ stands for a zero vector with appropriate dimension. The assumption (7) amounts to assuming $\mathbf{Q}_{n}$ block diagonal. The transition laws of the HMC (1) are thus Gaussian with:

$$
\begin{aligned}
p\left(\mathbf{x}_{n} \mid \mathbf{x}_{n-1}\right) & =\mathcal{N}_{\mathbf{x}_{n}}\left(\mathbf{f}_{n-1}\left(\mathbf{x}_{n-1}\right), \mathbf{Q}_{n-1}\right), \\
p\left(\mathbf{y}_{n} \mid \mathbf{x}_{n}\right) & =\mathcal{N}_{\mathbf{y}_{n}}\left(\mathbf{H}_{n} \mathbf{x}_{n}, \mathbf{R}_{n}\right),
\end{aligned}
$$

where $\mathcal{N}_{\mathbf{u}}(\mathbf{m}, \mathbf{C})$ denotes a Gaussian pdf with argument $\mathbf{u}$ and parameters $(\mathbf{m}, \mathbf{C})$.

\section{A. Sampling step (Prediction)}

Assume that one has an independently and identically distributed (i.i.d.) random set of samples (or particles), $\left\{\mathbf{x}_{n-1}^{k,(s)}\right\}_{s=1}^{S \gg 1}$, from $q\left(\mathbf{x}_{n-1}^{k} \mid \mathbf{y}_{0: n-1}\right)$, and wants to sample an i.i.d. random set, $\left\{\tilde{\mathbf{x}}_{n}^{k,(s)}\right\}_{s=1}^{S}$, from $q\left(\mathbf{x}_{n}^{k} \mid \mathbf{y}_{0: n-1}\right), k=$ $1, \cdots, K$. This can be achieved by first deriving (10) to provide an explicit form (but not exact) of $q\left(\mathbf{x}_{n}^{k} \mid \mathbf{x}_{n-1}^{k}, \mathbf{y}_{0: n-1}\right)$, then by using in (12) the (classical) hierarchical sampling technique [20, page 307].

1) Approximation of $q\left(\mathbf{x}_{n}^{k} \mid \mathbf{x}_{n-1}^{k}, \mathbf{y}_{0: n-1}\right)$ : From (14) we have,

$$
p\left(\mathbf{x}_{n}^{k} \mid \mathbf{x}_{n-1}\right)=\mathcal{N}_{\mathbf{x}_{n}^{k}}\left(\mathbf{f}_{n-1}^{k}\left(\mathbf{x}_{n-1}\right), \mathbf{Q}_{n-1}^{k}\right),
$$

where $\mathbf{f}_{n-1}^{k}\left(\mathbf{x}_{n-1}\right)$ denotes the $k^{\text {th }} n_{\mathbf{x}^{k}} \times 1$ sub-vector of $\mathbf{f}_{n-1}\left(\mathbf{x}_{n-1}\right)$, and $\mathbf{Q}_{n-1}^{k}$ the $k^{\text {th }} n_{\mathbf{x}_{n}^{k}} \times n_{\mathbf{x}_{n}^{k}}$ diagonal block of $\mathbf{Q}_{n-1}$. Inserting (16) in (10) shows that $q\left(\mathbf{x}_{n}^{k} \mid \mathbf{x}_{n-1}^{k}, \mathbf{y}_{0: n-1}\right)$ is Gaussian of mean,

$$
\boldsymbol{\mu}_{n}^{k}\left(\mathbf{x}_{n-1}^{k}\right)=\mathbb{E}_{q\left(\mathbf{x}_{n-1}^{k-} \mid \mathbf{y}_{0: n-1}\right)}\left[\mathbf{f}_{n-1}^{k}\left(\mathbf{x}_{n-1}\right)\right],
$$

${ }^{1}$ Without abuse of notation, the r.h.s. of (13) is rather proportional to an approximation of (5). and covariance, $\mathbf{Q}_{n-1}^{k}$. However, since the analytical computation of the mean (integral) (17) is not possible, we use the MC approximation of $q\left(d \mathbf{x}_{n-1}^{k-} \mid \mathbf{y}_{0: n-1}\right), \sum_{s=1}^{S} \frac{1}{S} \delta_{\mathbf{x}_{n-1}^{k-,(s)}}\left(d \mathbf{x}_{n-1}^{k-}\right)$, where $\delta_{\mathbf{u}}($.$) stands for the Dirac mass at point \mathbf{u}$, to approximate (17). One obtains,

$$
\hat{\boldsymbol{\mu}}_{n}^{k}\left(\mathbf{x}_{n-1}^{k}\right)=\frac{1}{S} \sum_{\ell=1}^{S} \mathbf{f}_{n-1}^{k}\left(\mathcal{X}_{k, n-1}^{\left(s_{\ell}\right)}\right),
$$

where $\quad \mathcal{X}_{k, n-1}^{\left(s_{\ell}\right)} \quad \stackrel{\text { def }}{=} \quad\left[\left(\mathbf{x}_{n-1}^{1,\left(s_{\ell}\right)}\right)^{T}, \cdots,\left(\mathbf{x}_{n-1}^{k-1,\left(s_{\ell}\right)}\right)^{T}\right.$, $\left.\left(\mathbf{x}_{n-1}^{k}\right)^{T},\left(\mathbf{x}_{n-1}^{k+1,\left(s_{\ell}\right)}\right)^{T}, \cdots,\left(\mathbf{x}_{n-1}^{K,\left(s_{\ell}\right)}\right)^{T}\right]^{T}, \quad$ and each subvector $\mathbf{x}_{n-1}^{j,\left(s_{\ell}\right)}$ with $j \neq k$ (all except the $k^{\text {th }}$ ) is drawn from the probability mass function (pmf), $\sum_{s=1}^{S} \frac{1}{S} \delta\left(\mathbf{x}_{n-1}^{j}-\mathbf{x}_{n-1}^{j,(s)}\right)$, where $\delta($.$) is the symbol of Kronecker.$

2) Computation of $\left\{\tilde{\mathbf{x}}_{n}^{k,(s)}\right\}_{s=1}^{S} \sim q\left(\mathbf{x}_{n}^{k} \mid \mathbf{y}_{0: n-1}\right)$ : Based on the previous result and (12), the hierarchical sampling technique can then be used to independently sample $\left\{\tilde{\mathbf{x}}_{n}^{k,(s)}\right\}_{s=1}^{S}$ from $q\left(\mathbf{x}_{n}^{k} \mid \mathbf{y}_{0: n-1}\right)$ by drawing each particle, $\tilde{\mathbf{x}}_{n}^{k,(s)}$, from the Gaussian, $\mathcal{N}\left(\hat{\boldsymbol{\mu}}_{n}^{k}\left(\mathbf{x}_{n-1}^{k,(s)}\right), \mathbf{Q}_{n-1}^{k}\right)$. The Sampling step of the VBMPF is summarized in Algorithm 1 below.

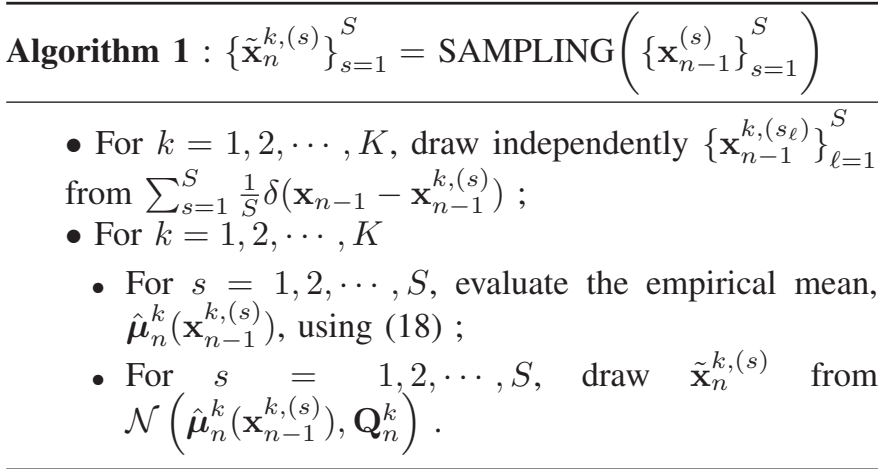

\section{B. Weighting and resampling steps (Filtering)}

We consider here a MC implementation of the Bayes' like formula (13). Starting from the i.i.d. prediction sets of particles, $\left\{\tilde{\mathbf{x}}_{n}^{k,(s)}\right\}_{s=1}^{S}, k=1,2, \cdots, K$, one wishes to sample i.i.d. sets of particles, $\left\{\mathbf{x}_{n}^{k,(s)}\right\}_{s=1}^{S}$, from $q\left(\mathbf{x}_{n}^{k} \mid \mathbf{y}_{0: n}\right)$, $k=1,2, \cdots, K$. Using the Rubin's Sampling Importance Resampling (SIR) strategy [21], this can be done by performing a weighting step assigning, for each $k$, a normalized weight, $\lambda_{n}^{k,(s)}$, followed by a resampling step from the discrete pmf, $\sum_{s=1}^{S} \lambda_{n}^{k,(s)} \delta\left(\mathbf{x}_{n}^{k}-\tilde{\mathbf{x}}_{n}^{k,(s)}\right)$.

Regarding the weighting step, one can show from (13) that for each $k$,

$$
\lambda_{n}^{k,(s)}=\frac{\mathcal{L}\left(\tilde{\mathbf{x}}_{n}^{k,(s)}\right)}{\sum_{s^{\prime}=1}^{S} \mathcal{L}\left(\tilde{\mathbf{x}}_{n}^{k,\left(s^{\prime}\right)}\right)} .
$$

Let $\mathbf{H}_{n}$ be divided into two parts, $\left(\mathbf{H}_{n}^{k}\right)_{n_{\mathbf{y}} \times n_{\mathbf{x}} k}$ and $\left(\mathbf{H}_{n}^{k-}\right)_{n_{\mathbf{y}} \times\left(n_{\mathbf{x}}-n_{\mathbf{x}^{k}}\right)}$, verifying:

$$
\mathbf{H}_{n} \mathbf{x}_{n}=\mathbf{H}_{n}^{k} \mathbf{x}_{n}^{k}+\mathbf{H}_{n}^{k-} \mathbf{x}_{n}^{k-} .
$$


Then, using (15), one can show that $\mathcal{L}\left(\widetilde{\mathbf{x}}_{n}^{k,(s)}\right)$ is expressed as

$$
\begin{aligned}
\mathcal{L}\left(\widetilde{\mathbf{x}}_{n}^{k,(s)}\right) & \propto \exp \left(-\frac{1}{2}\left[\left(\mathbf{H}_{n}^{k} \widetilde{\mathbf{x}}_{n}^{k,(s)}\right)^{T} \mathbf{R}_{n}^{-1} \mathbf{H}_{n}^{k} \widetilde{\mathbf{x}}_{n}^{k,(s)}\right.\right. \\
& \left.\left.-2\left(\mathbf{H}_{n}^{k} \widetilde{\mathbf{x}}_{n}^{k,(s)}\right)^{T} \mathbf{R}_{n}^{-1}\left(\mathbf{y}_{n}-\mathbf{H}_{n}^{k-} \overline{\mathbf{x}}_{n \mid n}^{k-}\right)\right]\right),
\end{aligned}
$$

where $\overline{\mathbf{x}}_{n \mid n}^{k-} \stackrel{\text { def }}{=} \mathbb{E}_{q\left(\mathbf{x}_{n}^{k-} \mid \mathbf{y}_{0: n}\right)}\left[\mathbf{x}_{n}^{k-}\right]$. One can also easily verify that $\mathcal{L}\left(\widetilde{\mathbf{x}}_{n}^{k,(s)}\right)$ is the value, at point $\mathbf{x}_{n}^{k}=\widetilde{\mathbf{x}}_{n}^{k,(s)}$, of the following Gaussian $\mathrm{pdf}^{2}$ :

$$
\mathcal{L}\left(\mathbf{x}_{n}^{k}\right)=\mathcal{N}_{\mathbf{x}_{n}^{k}}\left(\nu_{n}^{k}, \Sigma_{n}^{k}\right),
$$

where

$$
\begin{aligned}
\Sigma_{n}^{k} & =\left[\left(\mathbf{H}_{n}^{k}\right)^{T} \mathbf{R}_{n}^{-1} \mathbf{H}_{n}^{k}\right]^{-1}, \\
\nu_{n}^{k} & =\Sigma_{n}^{k}\left(\mathbf{H}_{n}^{k}\right)^{T} \mathbf{R}_{n}^{-1}\left[\mathbf{y}_{n}-\mathbf{H}_{n}^{k-} \overline{\mathbf{x}}_{n \mid n}^{k-}\right] .
\end{aligned}
$$

However, the computation of the mean, $\nu_{n}^{k}$, requires the knowledge of $\overline{\mathbf{x}}_{n \mid n}^{k-}=\mathbb{E}_{q\left(\mathbf{x}_{n}^{k-} \mid \mathbf{y}_{0: n}\right)}\left[\mathbf{x}_{n}^{k-}\right]$ (see (23)), which, in turn, is not known. Similarly to Section II-B, one way to overcome this is to approximate $\overline{\mathbf{x}}_{n \mid n}^{k-}$ by proceeding with iterations [16], [17], [19]. Nevertheless, we resort here to a non-iterative computationally efficient scheme by using the available prediction samples, $\left\{\tilde{\mathbf{x}}_{n}^{(s)}\right\}_{s=1}^{S}$. Our strategy consists in first computing a MC approximation of $\overline{\mathbf{x}}_{n \mid n} \stackrel{\text { def }}{=}$ $\mathbb{E}_{q\left(\mathbf{x}_{n} \mid \mathbf{y}_{0: n}\right)}\left[\mathbf{x}_{n}\right]$, then extracting the partition associated to $\overline{\mathbf{x}}_{n \mid n}^{k-}$. The MC approximation of $\overline{\mathbf{x}}_{n \mid n}$ is computed as follows:

$$
\begin{aligned}
\overline{\mathbf{x}}_{n \mid n} & =\int \mathbf{x}_{n} q\left(\mathbf{x}_{n} \mid \mathbf{y}_{0: n}\right) d \mathbf{x}_{n}, \\
& \approx \int \mathbf{x}_{n}\left[\frac{q\left(\mathbf{x}_{n} \mid \mathbf{y}_{0: n-1}\right) p\left(\mathbf{y}_{n} \mid \mathbf{x}_{n}\right)}{\int q\left(\mathbf{x}_{n} \mid \mathbf{y}_{0: n-1}\right) p\left(\mathbf{y}_{n} \mid \mathbf{x}_{n}\right) d \mathbf{x}_{n}}\right] d \mathbf{x}_{n} .
\end{aligned}
$$

Using the $\mathrm{MC}$ approximation, $q\left(\mathbf{x}_{n} \mid \mathbf{y}_{0: n-1}\right) \approx$ $\sum_{s=1}^{S} \frac{1}{S} \delta_{\widetilde{\mathbf{x}}_{n}^{(s)}}\left(d \mathbf{x}_{n}\right)$, in (25), one obtains:

$$
\overline{\mathbf{x}}_{n \mid n} \approx \sum_{s=1}^{S} \omega_{n}^{(s)} \widetilde{\mathbf{x}}_{n}^{(s)}
$$

where

$$
\begin{aligned}
\omega_{n}^{(s)} & \propto \mathcal{N}_{\mathbf{y}_{n}}\left(\mathbf{H}_{n} \widetilde{\mathbf{x}}_{n}^{(s)}, \mathbf{R}_{n}\right) ; \\
\sum_{s=1}^{S} \omega_{n}^{(s)} & =1 .
\end{aligned}
$$

Based on the weighted set of particles, $\left\{\tilde{\mathbf{x}}_{n}^{k,(s)}, \lambda_{n}^{k,(s)}\right\}_{s=1}^{S}$, any finite integral of the form $\int g\left(\mathbf{x}_{n}^{k}\right) q\left(\mathbf{x}_{n}^{k} \mid \mathbf{y}_{0: n}\right) d \mathbf{x}_{n}^{k}$ can be approximated by $\sum_{s=1}^{S} \lambda_{n}^{k,(s)} g\left(\tilde{\mathbf{x}}_{n}^{k,(s)}\right)$; the AM estimate of the $k^{\text {th }}$ partition, $\hat{\mathbf{x}}_{n \mid n}^{k}$, corresponds to $g\left(\mathbf{x}_{n}^{k}\right)=\mathbf{x}_{n}^{k}$. The weighting step of the VBMPF is summarized in Algorithm 2 below.

Once the weighted set of particles, $\left\{\tilde{\mathbf{x}}_{n}^{k,(s)}, \lambda_{n}^{k,(s)}\right\}_{s=1}^{S}$, is formed for each $k$, the resampling step is then performed

\footnotetext{
${ }^{2}$ To ensure that $\Sigma_{n}^{k}>\mathbf{0}$, one should have $n_{\mathbf{x}^{k}} \leq n_{\mathbf{y}}$ and $\left(\mathbf{H}_{n}^{k}\right)^{T}$ be full-rank. Nevertheless, if this is not verified, $\mathcal{L}\left(\mathbf{x}_{n}^{k}\right)$ is no longer a Gaussian pdf and (21)-(23) are no longer valid. In such a case, one can directly use (20) to compute the weights (19).
}

providing an i.i.d. set of particles, $\left\{\mathbf{x}_{n}^{k,(s)}, \frac{1}{S}\right\}_{s=1}^{S}$, by independently drawing each particle, $\mathbf{x}_{n}^{k,(s)}$, from the discrete pmf, $\sum_{s^{\prime}=1}^{S} \lambda_{n}^{k,\left(s^{\prime}\right)} \delta\left(\mathbf{x}_{n}^{k}-\tilde{\mathbf{x}}_{n}^{k,\left(s^{\prime}\right)}\right)$.

Algorithm 2: $\left\{\lambda_{n}^{k,(s)}\right\}_{s=1}^{S}=\operatorname{WEIGHTING}\left(\left\{\tilde{\mathbf{x}}_{n}^{(s)}, \frac{1}{S}\right\}_{s=1}^{S}\right)$

- For $s=1,2, \cdots, S$, use (27) to compute the weight, $\omega_{n}^{(s)}$, of the full joint particle, $\tilde{\mathbf{x}}_{n}^{(s)}$;

- Use (26) to compute a MC approximation of $\overline{\mathbf{x}}_{n \mid n}$. Those associated to $\overline{\mathbf{x}}_{n \mid n}^{k-}, k=1, \cdots, K$, can then be obtained by extraction;

- For $k=1,2, \cdots, K$, for $s=1,2, \cdots, S$, use (21)-(23) then (19) to compute the weight, $\lambda_{n}^{k,(s)}$, of the marginal particle, $\widetilde{\mathbf{x}}_{n}^{k,(s)}$. The AM estimate of the marginal state, $\mathbf{x}_{n}^{k}$, is then approximated by $\sum_{s=1}^{S} \lambda_{n}^{k,(s)} \widetilde{\mathbf{x}}_{n}^{k,(s)}$.

\section{Comments and remarks}

The proposed VBMPF algorithm combines VB-based density approximations with MC sampling techniques, in contrast with the classical MPFs which use MC sampling techniques only, based on the separability assumption of the (joint) filtering pdf, $p\left(\mathbf{x}_{n} \mid \mathbf{y}_{0: n}\right)$. Although the VBMPF shares the same sampling, weighting and resampling structure with the existing MPFs (see e.g. [7] [8]), it is nevertheless computationally more efficient since (i) the sampling step of these filters requires generating $J$ predicted particles (children) for each filtering particle, $\mathbf{x}_{n-1}^{k,(s)}$, (ii) computing their weights in the weighting step, and (iii) resampling a new set of particles. However, these filters still have the advantage of being applicable to systems for which the noises, $\mathbf{u}_{n}$ and $\mathbf{v}_{n}$, follow any type of distributions, not necessarily Gaussian. Note however that the proposed scheme can be extended to the case for which the factorization (7) of the HMC transition pdf is no longer valid (or equivalently, $\mathbf{Q}_{n}$ is no longer block diagonal), and to the case of a nonlinear measurement operator, $\mathbf{h}_{n}\left(\mathbf{x}_{n}\right)$, instead of $\mathbf{H}_{n} \mathbf{x}_{n}$.

It is generally difficult to clearly characterize the accuracy of VB-like approximations, since this may depend on several factors as for example the number of partitions, $K$, and the strength of dependencies between these partitions [15], [16], [22], [23]. Nevertheless, one can intuitively expect a good accuracy when the state partitions are weakly dependent a posteriori of each other. This can happen if each $k^{\text {th }}$ sub-vector $\mathbf{f}_{n}^{k}\left(\mathbf{x}_{n}\right)$ of the state transition function is weakly dependent of the other state sub-vectors, $\mathbf{x}_{n}^{k^{-}}$, the measurement matrix, $\mathbf{H}_{n}$, is nearly orthogonal, and the covariances, $\mathbf{Q}_{n}$ and $\mathbf{R}_{n}$, are block diagonal matrices whose diagonal blocks are $m \times m$ matrices, with $m \leq n_{\mathbf{x}^{k}}$. In contrast, when these partitions become strongly dependent, the "assumption" of conditional independence becomes too strong, and this may degrade the accuracy of the VB approximation. Consequently, the number of partitions $K$ should be chosen small enough to ensure best possible accuracy of VB-like approximations. However, the $\mathrm{MC}$ approximations, in turn, require larger number of particles, 
$S$, when the number of partitions, $K$, decreases (or, when the state partitions dimension, $n_{\mathbf{x}^{k}}$, increases). Thereby, for a given $S$, the guideline principle is to carefully choose $n_{\mathbf{x}^{k}}$ large enough (to achieve as accurate VB density estimate as possible), but not too large to mitigate the weights degeneracy phenomena.

\section{NUMERICAL SIMULATIONS}

We consider a semi-linear vectorial version of a state-space system, with length $N=50$, extensively used in the literature (see e.g. [5], [24]):

$$
\left\{\begin{array}{l}
x_{n+1}^{k}=0.5 x_{n}^{k}+\frac{25 \sum_{j=1}^{n_{\mathbf{x}}} x_{n}^{j}}{1+\left(\sum_{j=1}^{n_{\mathbf{x}}} x_{n}^{j}\right)^{2}}+8 \cos (1.2(n+1))+u_{n}^{k}, \\
y_{n}^{k}=0.5 x_{n}^{k}+v_{n}^{k}
\end{array}\right.
$$

$n_{\mathbf{x}}=n_{\mathbf{y}}=40, \mathbf{x}_{0} \sim \mathcal{N}(\mathbf{0}, 10 \times \mathbb{I}), \mathbf{u}_{n} \sim \mathcal{N}(\mathbf{0}, 10 \times \mathbf{I I})$ and $\mathbf{v}_{n} \sim \mathcal{N}(\mathbf{0}, \mathbf{R})$, where each $(i . j)^{\text {th }}$ entry of $\mathbf{R}$ is defined as:

$$
R(i, j)=\exp (-|i-j|) ; \quad i, j=1,2, \cdots, n_{\mathbf{y}} .
$$

We aim at assessing the performance of the VBMPF w.r.t. the $\mathrm{PF}$ [5] and the MPF [8] (which was - numerically - shown to be the most accurate among the class of MPFs [7]). We start by using the VBMPF and the MPF with full-factorization, i.e., the system state, $\mathbf{x}_{n}$, is partitioned into $K=n_{\mathbf{x}}$ (scalar) components, $x_{n}^{k}$. We use $S=10$ for the VBMPF and MPF (the number of children in MPF is $J=S$ ), and $S=10$ and $400\left(=10 \times n_{\mathbf{x}}\right)$ for the PF. Figure 1 plots the time-evolution of the MSE of the estimate of $\mathbf{x}_{n}$, averaged over 20 independent realizations of the system (with different initial states). As can be seen, the standard PF suffers from the large dimension of the system, requiring more than 400 particles to reach the accuracy of the MPF and VBMPF with only 10 particles. On the other hand, the proposed VBMPF is more performant than the MPF. This supports the relevance of using VB approach to insert independence on the joint filtering pdf, rather than a direct independence assumption.

We now investigate the sensitivity of the VBMPF and MPF to changes in the number of particles, $S$, and the dimension of state partitions, $n_{\mathbf{x}^{k}}$. For this purpose, we use the standard deviation defined by,

$$
\mathcal{D}=\frac{1}{N} \sum_{n=0}^{N-1}\left[\frac{1}{20} \sum_{j=1}^{20}\left\|\overline{\mathbf{x}}_{n \mid n}(j)-\mathbf{x}_{n}(j)\right\|^{2}\right]^{\frac{1}{2}},
$$

where $\mathbf{x}_{n}(j)$ is the state at the $j^{\text {th }}$ realization of the system, and $\overline{\mathbf{x}}_{n \mid n}(j)$ its estimate computed by one of the filtering algorithms ( $\mathcal{D}$ is averaged over 20 independent realizations, and $N=50$ time indices). Table I displays the standard deviation of the state estimates using the proposed VBMPF and the MPF with full-factorization, as a function of the number of particles. Overall, the performances of both filters improve with increased number of particles. One can further see that the VBMPF is more performant than the MPF for any number of particles, which is consistent with Figure 1.

Table II outlines the standard deviation of the state estimates using the proposed VBMPF and the MPF with $S=10$
TABLE I

STANDARD DEVIATION $\mathcal{D}$ OF THE STATE ESTIMATES USING THE VBMPF AND THE MPF WITH FULL-FACTORIZATION.

\begin{tabular}{|l||c|c|c|c|}
\hline$S$ & 5 & 10 & 15 & 20 \\
\hline \hline VBMPF & 16.3440 & 15.6697 & 15.4440 & 14.4968 \\
\hline MPF & 24.9437 & 23.2109 & 22.8409 & 21.9671 \\
\hline
\end{tabular}

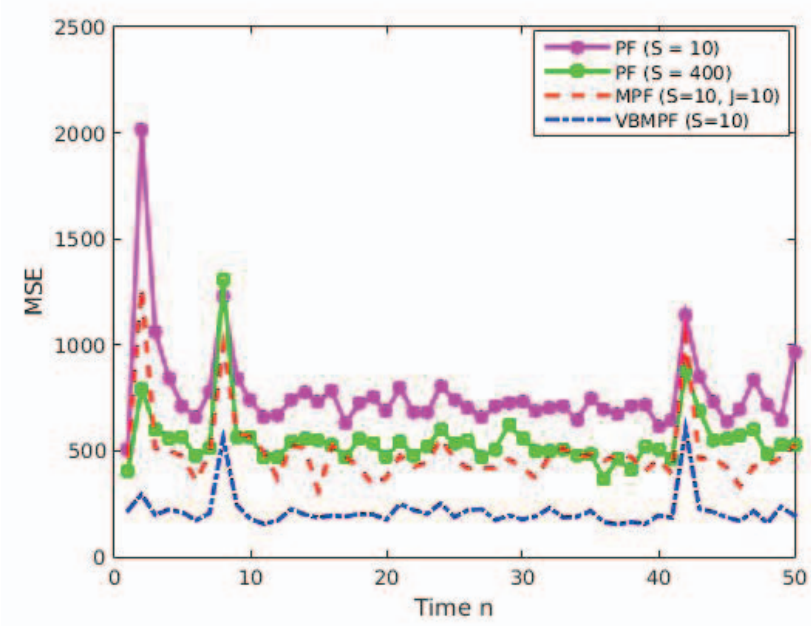

Fig. 1. MSE of estimation of $\mathbf{x}_{n}$ by PF, MPF and VBMPF with full factorization.

particles, as a function of the dimensions of partitions, $n_{\mathbf{x}^{k}}=$ $\frac{n_{\mathrm{x}}}{K}$. Overall, the performances of both filters degrade when increasing the dimension of partitions. This is of course due to the need of more particles to deal with the increase of $n_{\mathbf{x}^{k}}$. The proposed VBMPF behaves better than the MPF for small partitions $\left(n_{\mathbf{x}^{k}}=1,5\right)$, and worse for larger $\left(n_{\mathbf{x}^{k}} \geq 8\right)$. This can be explained by the fact that, on the one hand, 10 particles are not enough to sufficiently mitigate the degeneracy problem in the VBMPF for $n_{\mathbf{x}^{k}} \geq 8$, and on the other hand, the MPF actually uses more than 10 particles since each of these particles engenders 10 children in the sampling step (see Section III-C).

TABLE II

STANDARD DEVIATION $\mathcal{D}$ OF THE STATE ESTIMATES USING THE VBMPF AND THE MPF WITH $S=10$ PARTICLES.

\begin{tabular}{|l||c|c|c|c|c|}
\hline$n_{\mathbf{x}^{k}}$ & 1 & 5 & 8 & 10 & 20 \\
\hline \hline VBMPF & 15.6697 & 20.1057 & 23.4207 & 23.5091 & 27.4711 \\
\hline MPF & 23.2109 & 23.2223 & 23.3719 & 23.2047 & 24.0418 \\
\hline
\end{tabular}

\section{Conclusion}

We considered the Bayesian filtering problem for highdimensional systems. We first proposed a general framework based on the variational Bayesian (VB) approach approximating the filtering pdf of the full state as a separable product of marginal pdfs. We then used two classical random sampling 
techniques to derive Monte Carlo approximations of the obtained marginal distributions. In the proposed VB Multiple Particle Filter (VBMPF), the propagation of each particle requires generating one particle only, while in the existing MPF a set of (children) particles needs to be generated. The results of numerical experiments demonstrated the relevance of the VBMPF compared to the PF and MPF.

\section{REFERENCES}

[1] O. Cappé, E. Moulines, and T. Rydén, Inference in Hidden Markov Models, Springer-Verlag, 2005.

[2] M. Sanjeev Arulampalam, S. Maskell, N. Gordon, and T. Clapp, "A tutorial on particle filters for online nonlinear / non-Gaussian Bayesian tracking," IEEE Transactions on Signal Processing, vol. 50, no. 2, pp. 174-188, February 2002.

[3] H. Tanizaki, Nonlinear Filters, Estimation and Applications, Springer, Berlin, 2nd edition, 1996

[4] P.M. Djuric, J.H. Kotecha, A. Jianqui Zhang, A. YufeiHuang T. Ghirmai, and M.F. Bugallo nand J.Miguez, "Particle filtering," IEEE Signal Processing Magazine, vol. 20, no. 5, pp. 19-38, 2003.

[5] N. J. Gordon, D. J. Salmond, and A. F. M. Smith, "Novel approach to nonlinear/ non-Gaussian Bayesian state estimation," IEE Proceedings $F$, vol. 140, pp. 107-113, 1993.

[6] A. Doucet, N. de Freitas, and N. Gordon, Eds., Sequential Monte Carlo Methods in Practice, Statistics for Engineering and Information Science. Springer Verlag, New York, 2001.

[7] M.F. Bugallo and P.M. Djuric, "Particle Filtering for High-Dimensional Systems with Gaussian Approximations," in Proceedings of the IEEE International Conference on ICASSP, 2014.

[8] P.M. Djuric and M.F. Bugallo, "Particle Filtering for High-Dimensional Systems," in Proceedings of the IEEE International Workshop on CAMSAP, 2013.

[9] P.M. Djuric, T. Lu, and M.F. Bugallo, "Multiple Particle Filtering," in Proceedings of the IEEE International Conference on ICASSP, 2007.

[10] C. Snyder, T. Bengtsson, P. Bickel, and J. Anderson, "Obstacles To High-Dimensional Particle Filtering," Monthly Weather Review, vol. 136 , no. 12 , pp. 4629-40, 2008.

[11] T. Bengtsson, P.Bickel, , and B. Li, "Curse-of-dimensionality revisited: Collapse of the particle filter in very large scale systems," Probability and Statistics: Essays in Honor of David A. Freedman, vol. 2, pp. 31634, 2008.

[12] P.M. Djuric and M.F. Bugallo, "Multiple particle filtering with improved efficiency and performance," in Proceedings of the IEEE International Conference on ICASSP, 2015.

[13] M.F. Bugallo and P.M. Djuric, "Gaussian particle filtering in highdimensional systems," in Proceedings of the IEEE International Workshop on SSP, 2014.

[14] P. Closas and M.F. Bugallo, "Improving Accuracy by Iterated Multiple Particle Filtering," IEEE Signal Processing Letters, vol. 19, no. 8, pp. 531-34, 2012.

[15] T.S. Jaakkola, "Tutorial on variational approximation methods," in Advanced Mean Field Methods - Theory and Practice, M. Opper and D. Saad, Eds., pp. 129-59. MIT Press, 2001.

[16] V. Smidl and A. Quinn, The Variational Bayes Method in Signal Processing, Springer, 2006.

[17] V. Smidl and A. Quinn, "Variational Bayesian Filtering," IEEE Transactions on Signal Processing, vol. 56, no. 10, pp. 5020-5030, 2008.

[18] B. Ait-El-Fquih and T. Rodet, "Variational Bayesian Kalman Filtering in Dynamical Tomography," in Proceedings of the IEEE International Conference on Acoustics, Speech and Signal Processing (ICASSP), Prague, Czech Republic, May 2011.

[19] B. Ait-El-Fquih and I. Hoteit, "Fast Kalman-like Filtering in largedimensional linear and Gaussian state-space models," IEEE Transactions on Signal Processing, vol. 63, no. 21, pp. 5853-67, 2015.

[20] C. Robert, The Bayesian Choice: From Decision-Theoretic Foundations to Computational Implementation, Springer Science \& Business Media, New York, 2007.

[21] D.B. Rubin, "Using the SIR algorithm to simulate posterior distributions," in Bayesian Statistics, J.M. Bernardo, M.H. DeGroot, D.V. Lindley, and A.F.M. Smith, Eds., vol. 3, pp. 395-402. Oxford University Press, 1988.
[22] M.I. Jordan, Z. Ghahramani, T.S. Jaakkola, and L.K. Saul, "An introduction to variational methods for graphical models," Machine Learning, vol. 37, pp. 183-233, 1999.

[23] T.S. Jaakkola and M.I. Jordan, "Bayesian parameter estimation via variational methods," Statistics and Computing, vol. 10, pp. 25-37, 2000.

[24] F. Desbouvries, Y. Petetin, and B. Ait-El-Fquih, "Direct, Predictionand Smoothing-based Kalman and Particle Filter Algorithms," Signal Processing, vol. 91, no. 8, pp. 2064-2077, 2011. 